\title{
Fuzzy logic Controller Design for gun-turret system
}

\begin{abstract}
M. Galal*, N. G. Mikhail*, G. Elnashar*
Abstract: High precision control is desirable for future weapon systems. In this paper, several control design methodologies are applied to a weapon system to assess the applicability of each control design method and to characterize the achievable performance of the gun-turret system in precision control. The design objective of the gun-turret control system is to achieve a rapid and precise tracking response with respect to the turret motor command from the fire control system under the influences of disturbances, nonlinearities, and modeling uncertainties. A fuzzy scheme is proposed for control of multi-body, multi-input and multioutput nonlinear systems with joints represented by a gun turret-barrel model which consists of two subsystems: two motors driving two loads (turret and barrel) coupled by nonlinear dynamics. Fuzzy control schemes are employed for compensation and nonlinear feedback control laws are used for control of nonlinear dynamics. Fuzzy logic control (FLC) provides an effective means of capturing the approximate, inexact nature of the real world, and to address unexpected parameter variations and anomalies. Viewed in this perspective, the essential part of the FLC is a set of linguistic control rules related by the dual concepts of fuzzy implication and the compositional rule of inference. In essence, the FLC provides an algorithm which can convert the linguistic control strategy based on expert knowledge into an automatic control strategy. Accordingly, the design must be robust, adaptive, and, hopefully, intelligent in order to accommodate these uncertainties. Simulation results verify the desired system tracking performance.
\end{abstract}

Keywords: Multivariable nonlinear systems; weapon gun turret system; fuzzy logic control.

\section{Introduction}

Dc motors used in many applications such as steel rolling mills, electric trains, and robotic manipulators require speed controllers to perform tasks. Major problems in applying a conventional control algorithm as a speed controller are the effects of the nonlinearity in the system to be controlled. The nonlinear characteristics of the Dc motor such as saturation and friction could degrade the performance of the conventional controllers [1-3]. Many advanced model-based control methods such as variable-structure control [4] and model reference adaptive control [5] have been developed to reduce these effects. However, the performance of these methods depends on the accuracy of the system models and parameters. Generally, an accurate nonlinear model of an actual Dc motor is difficult to find, and parameter values obtained from system identification may be only approximated values.

\footnotetext{
${ }^{*}$ Egyptian Armed Forces
} 
Emerging intelligent techniques have been developed and extensively used to improve or to replace conventional control techniques because these techniques do not require a precise model. One of intelligent techniques, fuzzy logic developed by Zadeh [6, 7] is applied for controller design in many applications [8, 9]. A fuzzy logic controller (FLC) was proved analytically to be equivalent to a nonlinear PI controller when a nonlinear defuzzification method is used [10]. Also, the results from the comparison of conventional and fuzzy logic control techniques in the form of FLC [11, 12] and fuzzy compensator [13, 14] showed fuzzy logic can reduce the effects of nonlinearity in a DC motor and improve the performance of a controller. A FLC has been implemented on many platforms such as digital signal processor (DSP) [15], PC [16], or off-the-shelf microcontroller [17]. These platforms have different advantages and disadvantages. The FLC developed on DSP or PC can quickly process fuzzy computation to generate control efforts, but the physical size of the system may be too big and quite expensive for a small DC motor applications. On the other hands, using an off-the -shelf microcontroller to implement a FLC is inexpensive and the physical size of the system is small, but the FLC requires longer processing time. One way to improve the response time in microcontroller implementation approach is to use a look up table, but this method needs much more memory to store a table.

An alternative method to implement a FLC is using a fuzzy logic chip. The fuzzy logic chip is first developed in 1985 by Togai and Watanable [18]. It has been developed and improved continuously to be a commercial fuzzy logic microcontroller by many companies. The main feature of this chip is its capability in hardware level to execute fuzzy computation. Fuzzy rules and member ship functions are defined and stored in RAM or ROM by specific formats that make a designer's job easier. This feature could reduce developing time and bypass the need of a high speed yet expensive system to develop a FLC. The designer can also utilize other features included in a fuzzy logic microcontroller to reduce the size and to improve the system performance.

In this paper, a FLC is implemented for a position control of a Turntable and an Arm fixed on it each is derived by a DC motor. Heuristic knowledge is applied to define fuzzy membership functions and rules. The membership functions and rules are modified after initially borrowing the knowledge from a PI controller developed from a simple linear model [11, 14].

\section{System Description}

The gun-turret system shown in Fig. 1 consists of two revolute joints with two electric DC motors. The arm of length " $\mathrm{L}$ " is attached to the motor number " 2 ".

According to Dinavit_Hartinberg convention the coordinate frames are considered and rearranged as shown in figure. The joint parameters and the transformation matrices can be defined as shown in Table 1.

Table 1 Joint Parameters

\begin{tabular}{|l|llll|}
\hline Joint & $\Theta$ & $\mathrm{d}$ & $\mathrm{a}$ & $\alpha$ \\
\hline 1 & $\Theta_{1}$ & 0 & 0 & 90 \\
$1^{\prime}$ & 0 & 0 & $\mathrm{a}$ & 0 \\
2 & $\Theta_{2}$ & 0 & $\mathrm{~L}$ & 0 \\
\hline
\end{tabular}




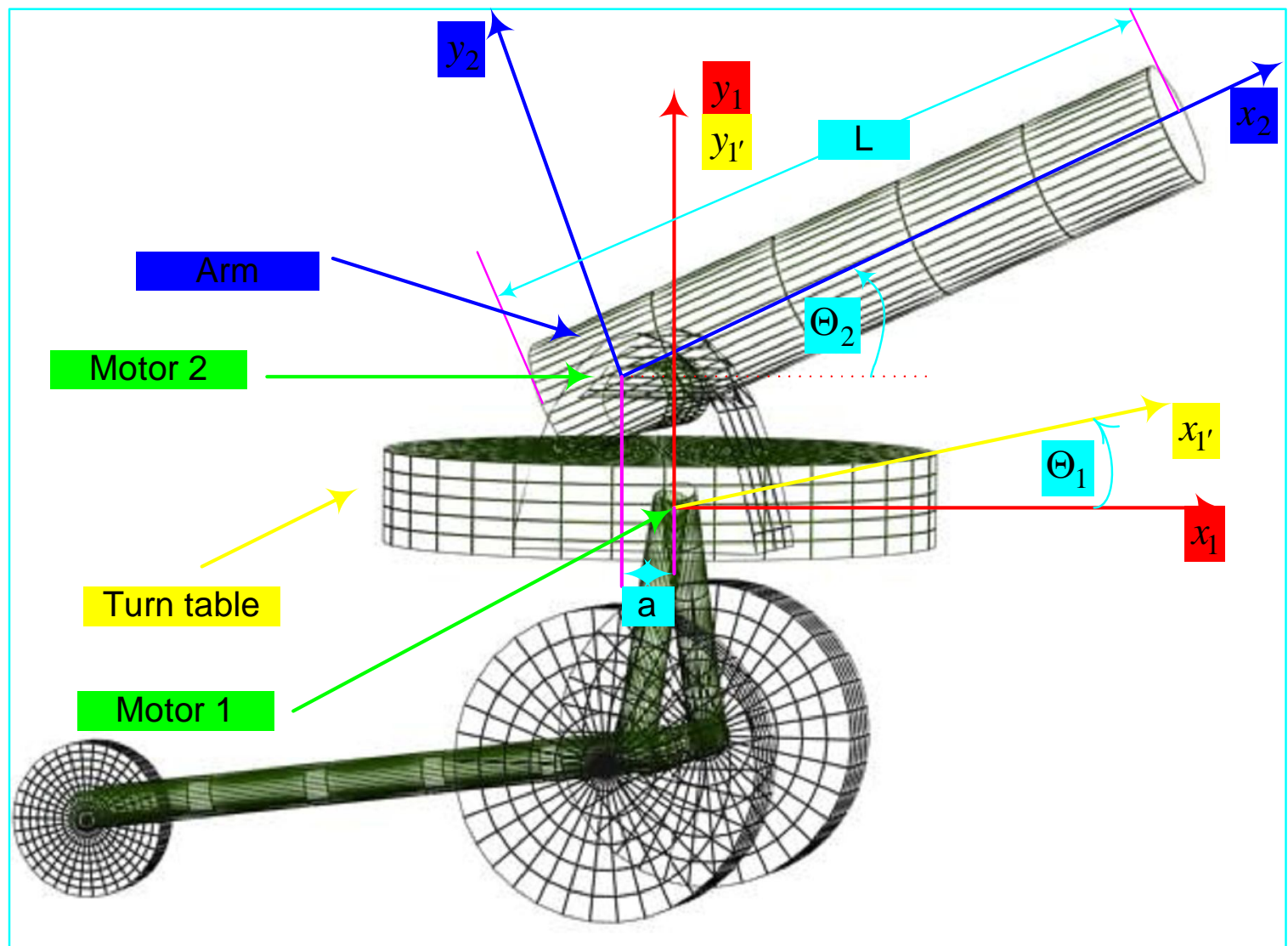

Fig. 1 The gun-turret system

Where the joint parameters $\Theta, \mathrm{d}$, a, and $\alpha$ are defined earlier with DH convention using the transformation matrix.

Lagrange's equation of motion (equation (1)) is applied to give two equations of motion, one to describe the turntable motion by angular coordinate $\Theta_{1}$ equation (11) and the second one to describe the motion of the arm by angular coordinate $\Theta_{2}$ equation (12).

$F=C_{F} P_{c} A_{t}$

$\frac{d}{d t}\left(\frac{\partial T}{\partial \dot{q}_{i}}\right)-\frac{\partial T}{\partial q_{i}}+\frac{\partial V}{\partial q_{i}}+\frac{\partial D}{\partial \dot{q}_{i}}=\mathrm{Q}_{i}$

where:

T ..... Total kinetic energy of the system.

$\mathrm{V}$...... Total potential energy of the system.

D ..... Dissipation function due to damping.

$\mathrm{Q}_{i} \ldots$ Generalized force corresponding to i-coordinate.

Then, the total kinetic energy, potential energy, dissipation function (if we have damping) and the virtual work of the system must be obtained to apply Lagrangian dynamic modeling. 


\section{Kinetic Energy}

The total kinetic energy of the system consists of four main terms, the kinetic energy of the $\operatorname{arm} \mathrm{T}_{A}$, the kinetic energy of the two rotors of the motors $\mathrm{T}_{R_{1}}$ and $\mathrm{T}_{R_{2}}$, and the kinetic energy of the payload at the end effector of the arm $\mathrm{T}_{p}$.

$\mathrm{T}=\mathrm{T}_{A}+\mathrm{T}_{R_{1}}+\mathrm{T}_{R_{2}}+\mathrm{T}_{p}$

The kinetic energy of the arm is due to the distribution of its mass along the length " $L "$ is defined by:

$\mathrm{T}_{A}=\frac{1}{2}(\rho \mathrm{AL}) \frac{L^{2}}{3} \dot{\Theta}_{2}^{2}+\frac{1}{2}(\rho \mathrm{AL})\left(\frac{L^{2}}{3} \mathrm{c}_{2}^{2}+\mathrm{a} \mathrm{L} \mathrm{c}_{2}+\mathrm{a}^{2}\right) \dot{\Theta}_{1}^{2}$

where

$\begin{array}{lll}\text { A } & \ldots \ldots \ldots & \text { Cross-section area. } \\ \rho & \cdots \ldots \ldots & \text { Density of the arm material }\end{array}$

$\mathrm{T}_{R_{1}}=\frac{1}{2} \mathrm{I}_{R_{1}} \Theta_{1}^{2}$

where

$\mathrm{T}_{R_{1}} \ldots \ldots \ldots$ Kinetic energy of rotor " 1 ".

$\mathrm{I}_{R_{1}} \ldots \ldots . \quad$ Mass moment of inertia of rotor " 1 ".

$\mathrm{T}_{R_{2}}=\frac{1}{2} \mathrm{I}_{R_{2}} \dot{\Theta}_{2}{ }^{2}+\frac{1}{2}\left(\mathrm{I}_{R_{2}}+\mathrm{m}_{R_{2}} \mathrm{a}^{2}\right) \dot{\Theta}_{1}^{2}$

where

$\mathrm{T}_{R_{2}} \quad \ldots . . . \quad$ Kinetic energy of rotor " 2 ".

$\mathrm{I}_{R 2} \quad \ldots \ldots . \quad$ Mass moment of inertia of rotor " 2 ".

$\mathrm{m}_{R_{2}} \quad \ldots \ldots . . \quad$ Mass of rotor " 2 ".

$\mathrm{T}_{p}=\frac{1}{2} \mathrm{~m}_{p}\left(\mathrm{LC}_{2}+\mathrm{a}\right)^{2} \dot{\Theta}_{1}^{2}+\frac{1}{2} \mathrm{~m}_{p} \mathrm{~L}^{2} \dot{\Theta}_{2}{ }^{2}$

where

$\mathrm{m}_{p}$......... Mass of the payload.

\section{Potential Energy}

The potential energy due to gravity of the arm, the payload and motor " 2 " represent the total potential energy of the system as the strain energy of the arm is neglected due to it is considered as a rigid body. 
- Gravitational potential energy of the arm

$$
\begin{aligned}
& \mathrm{V}_{A}=\int_{A r m} \mathrm{r}_{2}^{T} \mathrm{~g} \mathrm{dm} \\
& \mathbf{V}_{A}=-9.81 \rho \mathrm{A} \frac{L^{2}}{2} \mathrm{~S}_{2}
\end{aligned}
$$

- Gravitational potential energy of the payload

$$
\begin{aligned}
& \mathrm{V}_{p}=\mathrm{m}_{p} \underline{\mathrm{r}}_{p}^{T} \mathrm{~g} \\
& \mathbf{V}_{p}=-9.81 \mathrm{~m}_{p} \mathrm{LS}_{2}
\end{aligned}
$$

From equations (7) and (8), the total potential energy is defined as,

$$
\begin{aligned}
& \mathrm{V}=\mathrm{V}_{A}+\mathrm{V}_{p} \\
& \mathrm{~V}=-9.81 \rho \mathrm{A} \frac{L^{2}}{2} \mathrm{~S}_{2}-9.81 \mathrm{~m}_{p} \mathrm{LS}_{2}
\end{aligned}
$$

For a system without any external applied force at the end effector, the generalized force can be obtained from the virtual work of the system,

$$
\delta \mathrm{W}=\delta \underline{\mathrm{q}}^{T} \underline{\mathrm{Q}}
$$

The virtual work of the considered system is given by:

$$
\delta \mathrm{W}=\mathrm{M}_{1} \delta \Theta_{1}+\mathrm{M}_{2} \delta \Theta_{2}
$$

Then,

$$
\mathrm{Q}=\left(\begin{array}{l}
M_{1} \\
M_{2}
\end{array}\right)
$$

where

Q $\quad \ldots . . . .$. the generalized force.

$\mathrm{M}_{1} \ldots \ldots .$. applied torque at motor " 1 ".

$\mathrm{M}_{2} \ldots \ldots \ldots$ applied torque at motor " 2 ".

\section{Turntable's Equation of Motion}

Driving each term of the Lagrange's equation of motion we get that:

$$
\begin{aligned}
& {\left[\left(\frac{\rho A L^{3}}{3}+\mathrm{m}_{p} \mathrm{~L}^{2}\right) \mathrm{C}_{2}^{2}+\left(\rho \mathrm{AL}^{2} \mathrm{a}+2 \mathrm{~m}_{p} \mathrm{La}\right) \mathrm{C}_{2}\right.} \\
& \left.+\left(\rho \mathrm{A} \mathrm{La}^{2}+\mathrm{I}_{R_{1}}+\mathrm{I}_{R_{2}}+\mathrm{m}_{R_{2}} \mathrm{a}^{2}+\mathrm{m}_{p} \mathrm{a}^{2}\right)\right] \ddot{\Theta}_{1} \\
& -\left[2\left(\frac{\rho A L^{3}}{3}+\mathrm{m}_{p} \mathrm{~L}^{2}\right) \mathrm{C}_{2} \mathrm{~S}_{2}+\left(\rho \mathrm{AL}^{2} \mathrm{a}+2 \mathrm{~m}_{p} \mathrm{La}\right) \mathrm{S}_{2}\right] \dot{\Theta}_{2} \dot{\Theta}_{1}=\mathrm{M}_{1}
\end{aligned}
$$




\section{Arm's Equation of Motion}

$$
\begin{aligned}
& \left(\frac{\rho A L^{3}}{3}+\mathrm{I}_{R_{2}}+\mathrm{m}_{p} \mathrm{~L}^{2}\right) \ddot{\Theta}_{2} \\
& +\left(\frac{\rho A L^{3}}{3} \mathrm{C}_{2} \mathrm{~S}_{2}+\mathrm{m}_{p} \mathrm{~L}^{2} \mathrm{C}_{2} \mathrm{~S}_{2}+\frac{\rho \mathrm{AL}^{2} a}{2} \mathrm{~S}_{2}+\mathrm{m}_{p} \mathrm{LaS}_{2}\right) \Theta_{1}^{2} \\
& -9.81\left(\frac{\rho \mathrm{AL}}{2}+\mathrm{m}_{p} \mathrm{~L}\right) \mathrm{C}_{2}=\mathrm{M}_{2}
\end{aligned}
$$

where:

$$
\begin{array}{llll}
\Theta_{1}, \Theta_{2} & \ldots & \text { angular position of motor " } 1 \text { " and motor " } 2 \text { " respectively. } \\
c_{1}, s_{1} & \ldots & \cos \Theta_{1} \text { and } \sin \Theta_{1} \text { respectively. } \\
c_{2}, s_{2} & \ldots & \cos \Theta_{2} \text { and } \sin \Theta_{2} \text { respectively. } \\
\mathrm{L} & \ldots & \text { the arm length. } \\
\text { a } & \ldots & \text { the distance between the two motors in the } \mathrm{x}_{1} \text { direction. }
\end{array}
$$

\section{Controller Design}

While conventional controllers depend on the accuracy of the system model and parameters, FLCs use a different approach to control the system. Instead of using system model, the operation of a FLC is based in heuristic knowledge and linguistic descriptions to perform a task. The effects from inaccurate parameters and models are reduced because a FLC does not require a system model. However, building a FLC from the ground-up may not provide good result than a conventional controller if there is not enough knowledge of the system. Therefore, in this paper, the FLC is initially constructed as a-priori knowledge in the process. The performance of the FLC is then improved by adjusting the results and membership functions. These design procedure are described as follows.

\section{Procedure 1: Defining inputs, outputs, and universe of discourse}

To apply heuristic knowledge in the FLC, inputs, outputs, and universe of discourse are defined first. The inputs are the error $(\mathrm{E})$ between the reference position $\left(\Theta_{r}\right)$ and the actual position $\left(\Theta_{a}\right)$, and the change in error $(\mathrm{CE})$. The output is the change in armature voltage (CU). The inputs and output illustrated in Fig. 2 are described by:

$\mathrm{E}=\mathrm{e}(\mathrm{k})=\Theta_{r}(\mathrm{k})-\Theta_{a}(\mathrm{k})$

$\mathrm{CE}=\mathrm{e}(\mathrm{k})-\mathrm{e}(\mathrm{k}-1)$

$\mathrm{CU}=\mathrm{u}(\mathrm{k})-\mathrm{u}(\mathrm{k}-1)$

where

$\mathrm{K} \quad \ldots$ is the time index. 


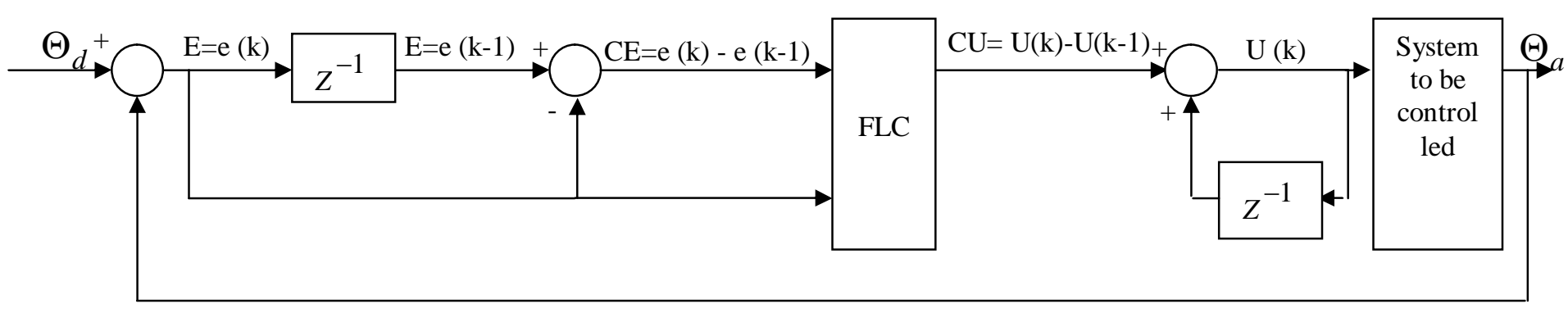

Fig. 2 Block diagram of the FLC

The maximum range of the system angular position is $+/-60^{\circ}$. The possible error in the range is between $-120^{\circ}$ to $+120^{\circ}$. Therefore, the universe of discourse of $\mathrm{E}$ is defined to span between $-120^{\circ}$ to $+120^{\circ}$. The universe of discourse of the change in error is based on the experiment data from the PI controller design included in procedure 2, which gives the range of error change, is $+/-5.5^{\circ}$. For the change in armature voltage, the minimum and maximum defined value are $-1.5 \mathrm{~V}$ and $+1.5 \mathrm{~V}$ respectively.

\section{Procedure 2: Defining fuzzy membership functions and rules}

To perform fuzzy computation, the inputs and output must be converted from numerical or "crisp" value into linguistic forms. The terms such as "Small" and "Big" are used to quantize the inputs and output values to linguistic terms that used to represent the input and output values are defined by seven fuzzy variables as shown in table 2 .

Table 2 Fuzzy linguistic terms

\begin{tabular}{|c|c|c|c|c|c|c|c|}
\hline Term & PB & PM & PS & ZE & NS & NM & NB \\
\hline \multirow{2}{*}{ Definition } & Positive & Positive & Positive & Zero & Negative & Negative & Negative \\
& Big & Medium & Small & & Small & Medium & Big \\
\hline
\end{tabular}

Fuzzy membership functions are used as tools to convert crisp values to linguistic terms forms. A fuzzy membership function can contain several fuzzy sets depending on how many linguistic terms. In this paper, seven fuzzy sets are obtained by applying the seven linguistic terms. The number for indicating how much a crisp value can be a member in each fuzzy set is called a degree of membership. One crisp value can be converted to be partly in many fuzzy sets, but the membership degree in each fuzzy set may be different.

In order to define fuzzy membership function, designers can choose many different shapes based on their preference or experience. The popular shapes are triangular and trapezoidal because these shapes are easy to represent designer's ideas and require low computation time. For performing fine-tuning to improve the efficient of the controller, the adjacent of each fuzzy set value should overlap about $25 \%$ [19]. The initial membership functions are illustrated in Fig. 3. 
In equations $(2,3)$, we see that the equations of motion are in a nonlinear form. We ought to use FLC to overcome this nonlinearity of the equation of motion of the system to be controlled.

Instead of using mathematical formulas, a FLC uses fuzzy rules to make a decision and generate the control effort. The rules are in the form of IF-THEN statements. For example, IF the error $(\mathrm{E})$ is equal to Positive Big (PB) and the change in error (CE) is equal to Positive Medium (PM) THEN the change in armature voltage (CU) is equal to Negative Medium (NM). The matters in defining rules are how many rules should be used and how to determine the relation in IF-THEN statements. Actually, the solutions are based on the experience of a designer or the previous knowledge of the system. The critical point is if there is not sufficient knowledge applied in the design, the result could be drastically bad.

The initial rules are constructed as shown in Table 3 . The efficiency can be improved by adjusting the membership functions and rules in procedure 3.

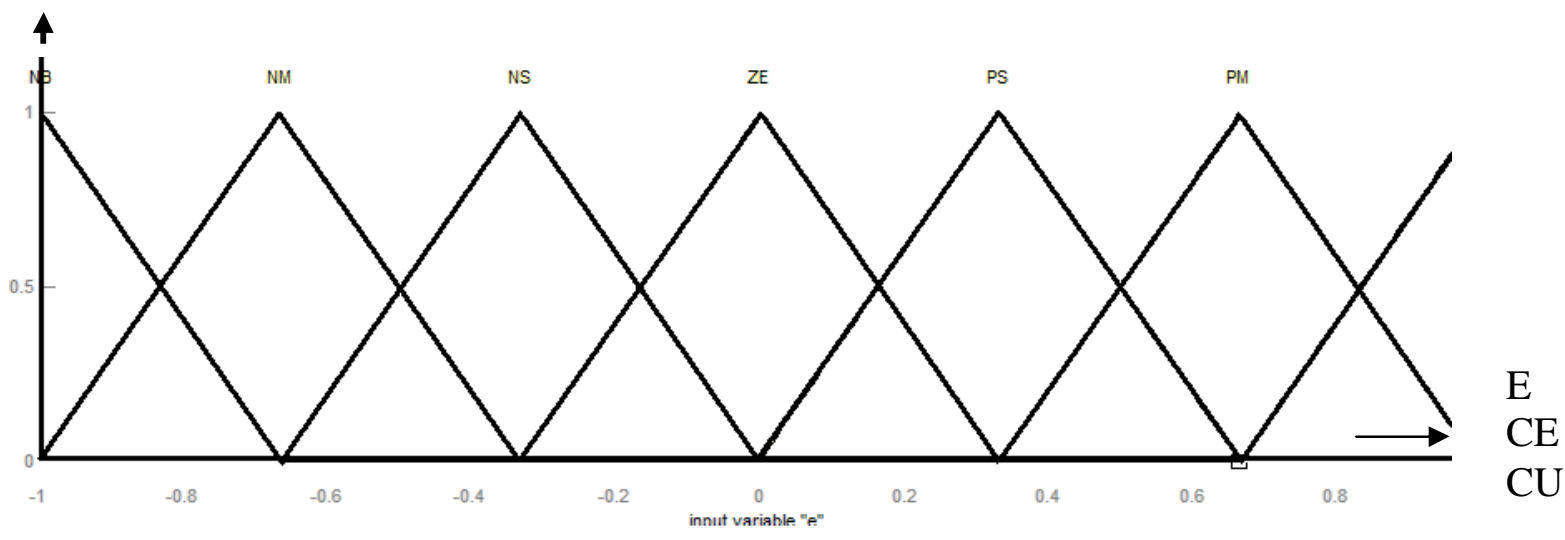

Fig. 3 Initial membership functions.

Table 3 Initial rules

\begin{tabular}{|c|ccccccc|}
\hline ce & NB & NM & NS & ZE & PS & PM & PB \\
\hline NB & NB & NB & NB & NB & NM & NS & ZE \\
NM & NB & NB & NB & NM & NS & ZE & PS \\
NS & NB & NB & NM & NS & ZE & PS & PM \\
ZE & NB & NM & NS & ZE & PS & PM & PB \\
PS & NM & NS & ZE & PS & PM & PB & PB \\
PM & NS & ZE & PS & PM & PB & PB & PB \\
PB & ZE & PS & PM & PB & PB & PB & PB \\
\hline
\end{tabular}

To send out the armature voltage output, the output in the form of fuzzy sets must be converted to a crisp value. This process is called defuzzification. In this paper, the center of gravity method is chosen. The formula of this method is

$\mathrm{Z}=\frac{\sum_{i=1}^{n} S_{i} F_{i}}{\sum_{i=1}^{n} F_{i}}$ 
where $\mathrm{Z}$ is the output from defuzzification, $\mathrm{S}_{i}$ is the specific position at the $\mathrm{i}^{\text {th }}$ is the fuzzy set, and $\mathrm{F}_{i}$ is the membership degree at that position.

\section{Procedure 3: Adjusting fuzzy membership functions and rules}

In order to improve the performance of the FLC, the rules and membership functions are adjusted by making the area of membership functions near ZE region narrower to produce finer control resolution. On the other hands, making the area far from ZE region wider gives faster control response. Also, the performance can be improved by changing the severity of the rules [11]. After adjusting the rules and membership functions, the final rules and membership functions are obtained as shown in, Table 4 and Fig. 4 respectively.

Table 4 Modified rules

\begin{tabular}{|c|ccccccc|}
\hline ce & NB & NM & NS & ZE & PS & PM & PB \\
\hline NB & NB & NB & NB & NB & ZE & PM & PM \\
NM & NB & NB & NB & NM & PS & PM & PB \\
NS & NB & NB & NM & NS & PM & PM & PB \\
ZE & NB & NM & NS & ZE & PB & PB & PB \\
PS & NM & NS & ZE & PS & PB & PB & PB \\
PM & NS & ZE & PS & PM & PB & PB & PB \\
PB & ZE & PS & PM & PB & PB & PB & PB \\
\hline
\end{tabular}

Observing the performance of the system after applying the modified rules we get that there are some unused rules and in order to reduce memory size and have a faster performance we ought to eliminate the unused rules, so we get only 33 rules, table (5) shows the reduced modified rules.

Table 5 Reduced Modified Rules

\begin{tabular}{|c|ccccc|}
\hline ce & NB & NM & NS & ZE & PS \\
\hline NB & NB & NB & NB & NB & ZE \\
NM & NB & NB & NB & NM & PS \\
NS & NB & NB & NM & NS & PM \\
ZE & NB & NM & NS & ZE & PB \\
PS & NM & NS & ZE & PS & PB \\
PM & NS & ZE & PS & PM & -- \\
PB & ZE & PS & PM & PB & -- \\
\hline
\end{tabular}



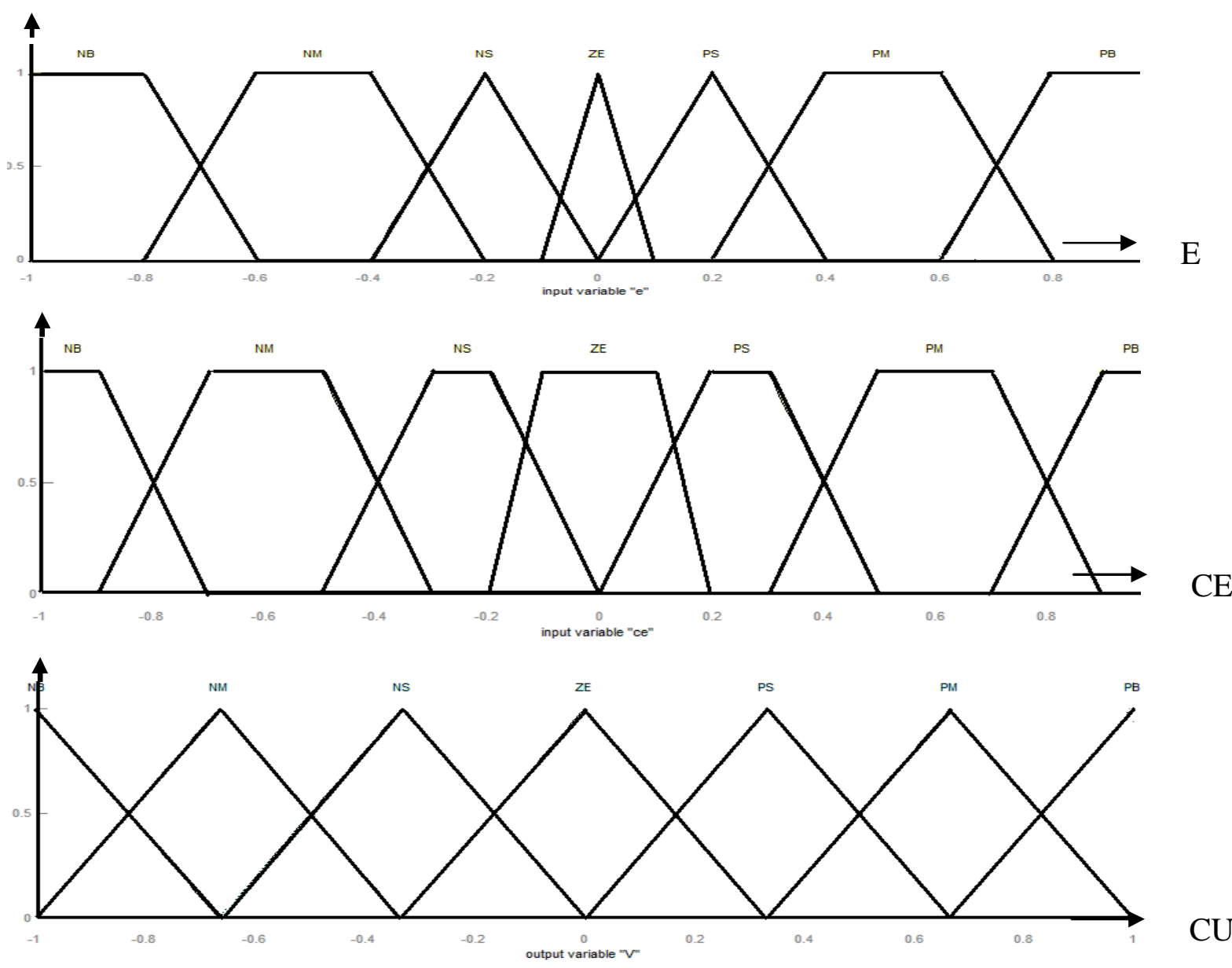

Fig. 4 Modified membership functions

\section{Simulation Results}

Matlab / Simulink software is used to simulate the Gun equation of motion and to make a good comparison between performances of FLC with 49 rules before and after adjusting the fuzzy membership functions and rules, step and half wave sinus signals were applied to the system as a control inputs. Both the controllers' step responses of the system were given in Fig. 5.a. For this work, rising times and overshot according to the modified FLC are better than in original the one which it's performance is out of the control aim.

\section{Conclusions}

This paper developed a solution to control of a two motor load system using fuzzy logic control. The implementation procedure of advanced control algorithms in the MATLAB/ SIMULINK software environment is presented to execute the control of the gun turret control system; and the results have shown that the developed control system is valid for advanced weapon control. Also, the designed control system can be applied to other complex controlled plants. The level of performance for each control algorithm is based on how well each they measure up to the criteria developed from the system. Simulation results show that modifying FLC responds with less overshot and minimum settling time. The modified controller showed a good position tracking performance. 


\section{References}

[1] B. J. Chalmers, "Influence of saturation in brushless permanent-magnet motor drives," IEE Proc. B, Electr. Power Appl., vol. 139, no. 1, pp.51-52, 1992.

[2] C. T. Johnson and R.D. Lorenz, "Experimental identification of friction and its compensation in precise, position controlled mechanics," IEEE Trans. Ind. Applicant., vol. 28, no. 6, pp. 1392-1398, 1992.

[3] C. Canudas, K. J. Astrom, and K. Braun, "Adaptive friction compensation in DC-motor drives,” IEEE J. Robot., Automat., vol. RA-3, no. 6, pp. 681-685, 1987.

[4] J. Y> Hung, W. Gao, and J. C. Hung, "Variable structure control: A survey," IEEE Trans. Ind. Electron., vol. 40, no. 1, pp. 2-22, 1993.

[5] H. Butler, G. Honderd, and J. V. Amerongen, "Model reference adaptive control of a direct-drive DC motor," IEEE Mag. Contr. Sys., vol. 9, no. 1, pp.80-84, 1989.

[6] L. A. Zadeh, "Fuzzy sets," Informat. Control, vol. 8, pp. 338-353, 1965.

[7] L. A. Zadeh, "Outline of a new approach to the analysis complex systems and decision processes," IEEE Trans. Syst. Man Cybern., vol. SMC-3, pp. 28-44,1973.

[8] M. Chow and H. Tarm, "Application of fuzzy logic technology for spatial load forecasting,” IEEE Trans. Power Syst. Vol. 12, no. 3, pp. 1360-1366, 1997.

[9] M. Chow, J. Zhu and H. Tarm, "Application of fuzzy multi-objective decision making in spatial load forecasting," IEEE Trans. Power Syst. Vol. 13, no. 3, pp. 1185-1190, 1998.

[10] H. Ying, W. Siler, and J. J. Buckley, "Fuzzy control theory: A nonlinear case," Automatica, vol. 26, no. 3, pp. 513-520, 1990.

[11] M. Chow and A. Menozzi, "On the comparison of emerging and conventional techniques for DC motor control," Proc. IECON, pp. 1008-1013. 1992.

[12] J. H> Kim, J.H. Park, S. W. Lee, and e. K. P. Chong, "a two-layered fuzzy logic controller for systems with deadzones," IEEE Trans. Ind. Electron., vol. 41, no. 2, pp. 155-162,1994.

[13] J. H Kim, K. C Kim, and E. K. P. Chong, "Fuzzy precompensated PID controllers," IEEE Trans. Contr. Sys. Tech., vol. 2, n0. 4, pp. 406-411, 1994.

[14] J. T. Teeter, M. Chow, and J. J. Brickely Jr., "A novel fuzzy friction compensation approach to improve the performance of a DC motor control system," IEEE Trans. Ind. Electron., vol. 43, no. 1, pp. 113-120, 1996.

[15] C. S. Liu, J. C. Yang and J. S. Chen, "A normalized smoothing control scheme based on fuzzy modeling for the control of a DC brushless servo drives," Proc. AMC '96-MIE, vol. 1, pp. 29-24, 1996.

[16] P. I. Lin and C. Ellsworth, "Design and implementation of a PC-based universal fuzzy logic controller system," Int. Conf. IEEE/IAS, pp. 399-408, 1995.

[17] P. Guillemin, "Fuzzy logic applied motor control," IEEE Trans. Ind. Applicat. Vol. 32, no. 1, pp. 51-56, 1996.

[18] M. Togai and H. watanabe, "Expert system on a chip: An engine for real-time approximation reasoning,” IEEE Expert Syst. Mag., vol. 1, pp. 55-62, 1986.

[19] B. Kosko, Neural networks and fuzzy systems, Prentice Hall, 1991. 


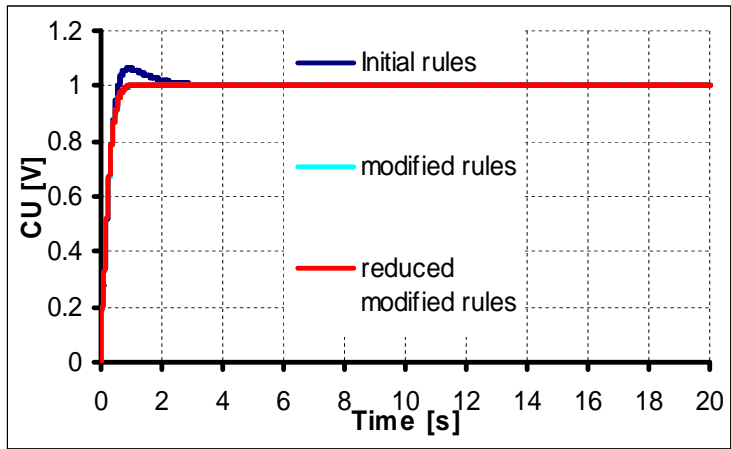

Fig. 5.a Step responses of the Arm under different controllers

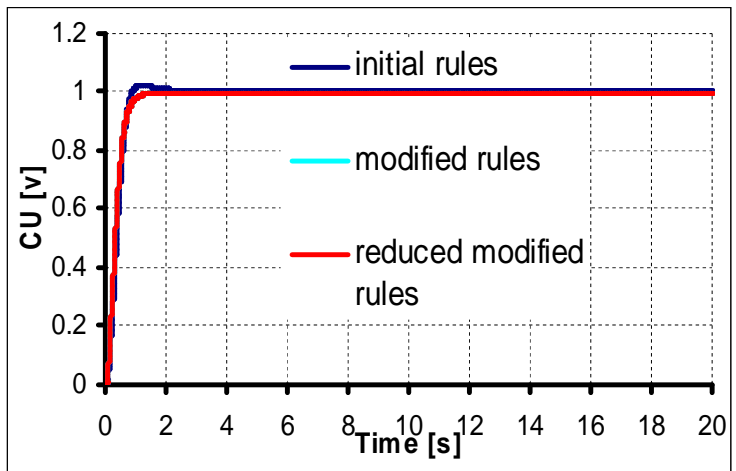

Fig. 5.c Step responses of the turntable under different controllers

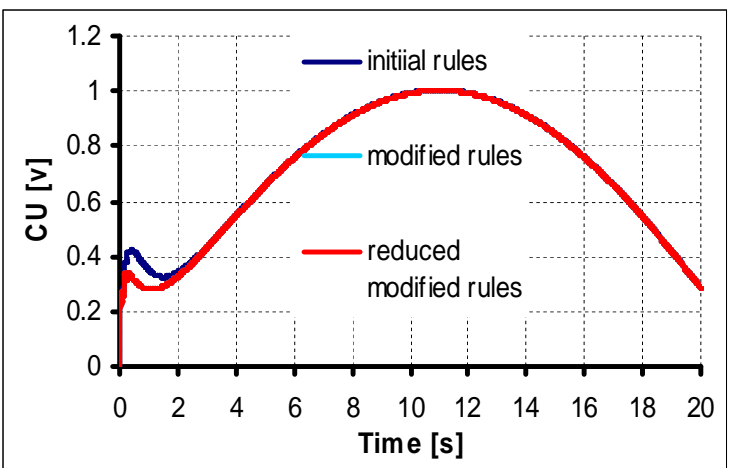

Fig. 5.b Sinus responses of the Arm under different controllers

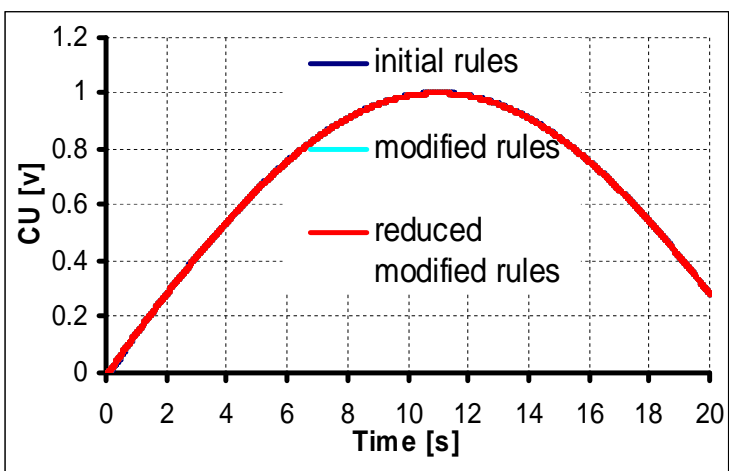

Fig. 5.d Sinus responses of the turntable under different controllers

Fig. 5 Step and Sinus System's responses under different controllers 